\title{
Tractive performance of Trelleborg PneuTrac tyres
}

\author{
Michele Mattetti, ${ }^{1}$ Massimiliano Varani, ${ }^{1}$ Mirko Maraldi, ${ }^{1}$ Francesco Paolini, ${ }^{2}$ Stefano Fiorati, ${ }^{2}$ \\ Giovanni Molari ${ }^{1}$
}

\author{
${ }^{1}$ Department of Agricultural and Food Sciences, Alma Mater Studiorum, University of Bologna; ${ }^{2}$ CNH Industrial - \\ Tractor Innovation Engineering, Modena, Italy
}

\begin{abstract}
In the last decades, heavier and more powerful tractors were introduced to the market and they require bigger tyres in order to exert higher traction forces but also to limit soil compaction. Therefore, different solutions were proposed by manufacturers to increase the footprints of traction elements, so that a higher drawbar pull is allowed especially in cohesive soils. However, these solutions have provided a limited increase in the traction efficiency. Recently, Trelleborg have developed a tyre named PneuTrac. The main feature of this tyre lies in the fact that the carcass is radially flexible like a standard radial tyre, but still able to support cornering loads like tracks. This allows the tyre to run with a very low inflating pressure. The aim of this paper was to compare the tractive performance of a set PneuTrac with that of an equivalent set of standard radial tyres. Both types of tyre were mounted on the same tractor, equipped with a CAN-Bus data logger, a load cell and a GPS receiver to measure the drawbar pull and other vehicle operating parameters. Drawbar tests were carried out in three different soil conditions. Results show that PneuTrac performance was slightly less affected by soil conditions than in the case of traditional radial tyres. Overall, PneuTrac tyres permit to increase the drawbar pull up to $5.7 \%$ and to reduce slip. PneuTrac tyres also provided a $7.7 \%$ increase in the power delivery efficiency with respect to traditional radial tyres.
\end{abstract}

Correspondence: Michele Mattetti, Department of Agricultural and Food Sciences (DISTAL), Alma Mater Studiorum, University of Bologna, via G. Fanin 50, 40127 Bologna, Italy.

Tel.: +39.051.2096174 - Fax: +39.051.2096178.

E-mail: michele.mattetti@unibo.it

Key words: Trelleborg PneuTrac; radial tyres; drawbar pull; power delivery efficiency; traction.

Acknowledgements: the Authors are grateful to Piero Mancinelli, Karel Štefekc, Claudia Di Dio for the provision of both tyre sets and Enrico Trevisi for his assistance during the field test activities.

Received for publication: 13 November 2019.

Accepted for publication: 2 April 2020.

(C) Copyright: the Author(s), 2020

Licensee PAGEPress, Italy

Journal of Agricultural Engineering 2020; LI:1031

doi:10.4081/jae.2020.1031

This article is distributed under the terms of the Creative Commons Attribution Noncommercial License (by-nc 4.0) which permits any noncommercial use, distribution, and reproduction in any medium, provided the original author(s) and source are credited.

\section{Introduction}

World population growth and low world market prices are pushing farms to increase their productivity and efficiency (Olesen and Bindi, 2002). Productivity and efficiency can be increased through more sophisticated and larger machines, therefore tractors are becoming more powerful and heavier, so that higher traction forces can be generated. Traction forces result in normal and shear stresses in the soil; normal stress depend upon the combination of tractor weight and traction element footprint, while shear stress depend upon soil shear strength, which in turn is a function of soil friction angle, soil cohesion, and normal stress as well. Shear stress determines soil longitudinal deformation, which is the major contributor to the vehicle slippage (McKyes, 2012). Vehicle slippage and motion resistance are responsible for the traction power losses, which ranges from 20 up to $50 \%$ of the engine power thrust depending on soil type and conditions, and vehicle configuration (Regazzi et al., 2019). Vehicle slippage can be decreased by increasing tractor weight or increasing the contact area between the soil and traction element. However, weight should be limited as much as possible, since it leads to higher soil compaction, that is detrimental for soil and crops (Schjønning et al., 2015). For these reasons, over the last decades, many solutions have been proposed with the aim of increasing the footprint of traction elements. These solutions are dual and triple-tyre configurations, belted rubber tracks, triangular rubber tracks for conventional tractors, low pressure tyres and tyres with larger diameters. All of these provide a higher traction performance especially in cohesive soil (McKyes, 2012). In terms of traction efficiency, dual-tyre configuration is more efficient than single-tyre configuration especially in very loose soil. On the other hand, triple-configuration is less efficient than both configurations, since the axle load is distributed among more tyres, which might be overinflated (Bashford, Von, et al., 1987; Turner, 1993). Belted rubber tracks and triangular tracks for conventional tractors tend to be more efficient than wheeled tractors mainly due to a lower slippage caused by the higher mass and longer contact patch; however, they are much heavier than wheels, therefore they lead to an extra soil load (Arvidsson et al., 2011; Molari, et al., 2012, 2015). Low pressure tyres are constituted of a more flexible carcass than normal tyres, so their footprint can be larger and their efficiency is slightly higher than that of normal tyres (Šmerda and Čupera, 2010). Another way to increase the tyre footprint is using tyres with a larger diameter; this solution allows to increase both drawbar pull and efficiency (Bashford et al., 1993).

Recently, Trelleborg has developed the PneuTrac tyre that will be described briefly in the paper. Further details can be found in the patent (Patent No. US20140158268 A1, 2014). The wheel assembly of the tyre has uneven flexibility. In particular, the tyre is circularly flexible, while keeping the radial rigidity. This is thanks to the curved sidewall, that it can naturally fold even if it is 
constituted of rigid elements. Thus, the footprint of PneuTrac tyres can flatten out and it is able to better adapt to the ground. Moreover, the wheel assembly is self-supporting, so air is not necessary for delivering traction forces and it supports cornering loads like tracks.

Thus, PneuTrac operate with lower inflating pressure than standard radial tyres and thus they can achieve larger footprints (Kumar et al., 2018). In the aerospace industry, a similar concept was adopted for the Exomars rover, which was fitted with flexible metal wheels instead of rigid ones. Such wheels are subjected to a significant deformation if loaded, so that their footprints can be very large. Especially on soft soil, they provide higher traction performance than rigid wheels of the same size and under the similar operating conditions (Patel et al., 2010).

For these reasons, similar improvements are expected for Trelleborg PneuTrac tyres with respect to standard radial tyres. The objective of this paper was the comparison of the tractive performance of Trelleborg PneuTrac with those of equivalent standard radial tyres.

\section{Materials and methods}

Tests were carried out with a Case IH Maxxum 115 (CNH Industrial N.V., Amsterdam, NL) in two different configurations, one with Trelleborg PneuTrac tyres and the other with standard radial tyres. In the following, these configurations are labelled $P T$ and $S R T$, respectively. Both tyre-sets were brand new and have the same thread design, so the performance differences are only due to the tyre structure. The specifications of both tractor configurations are reported in Table 1.

With respect to the standard radial tyres used in the study, each front PneuTrac tyre is $94 \mathrm{~kg}$ heavier (58\% heavier) than that of the standard radial tyre; while each rear PneuTrac tyre is $170 \mathrm{~kg}$ heavier $(62 \%$ heavier $)$ than that of the standard radial tyre. In order to maintain the same total mass between the two configurations, front and rear ballasts were fitted on the tractor for the SRT configure (Figure 1). This led to a slightly different (2\%) mass distribution between the two configurations, however, recent studies demonstrated that the impact of weight distribution on tractive performance is much lower than that of the tractor mass (Varani et al., 2018; Regazzi et al., 2019). Tyre pressures were set in order to achieve the lowest recommended inflating pressure for the wheel load and to match the load capacities of tyres of the same axle. This solution was chosen because, it leads to highest tractive performance (Brassart, 1994). PneuTrac rolling radius is $2.6 \%$ smaller than that of standard tyres and this resulted in a lower engine-to-wheel ratio which led to a lower maximum ground speed for PT. PneuTrac are characterized by a larger footprint, caused by a longer rather than wider contact patch. Tyre footprints were measured according to the method reported by Kumar (2018). Moreover, the lead was calculated from the distance travelled by the tractor, with the front axle disabled and enabled, during 10 rev-

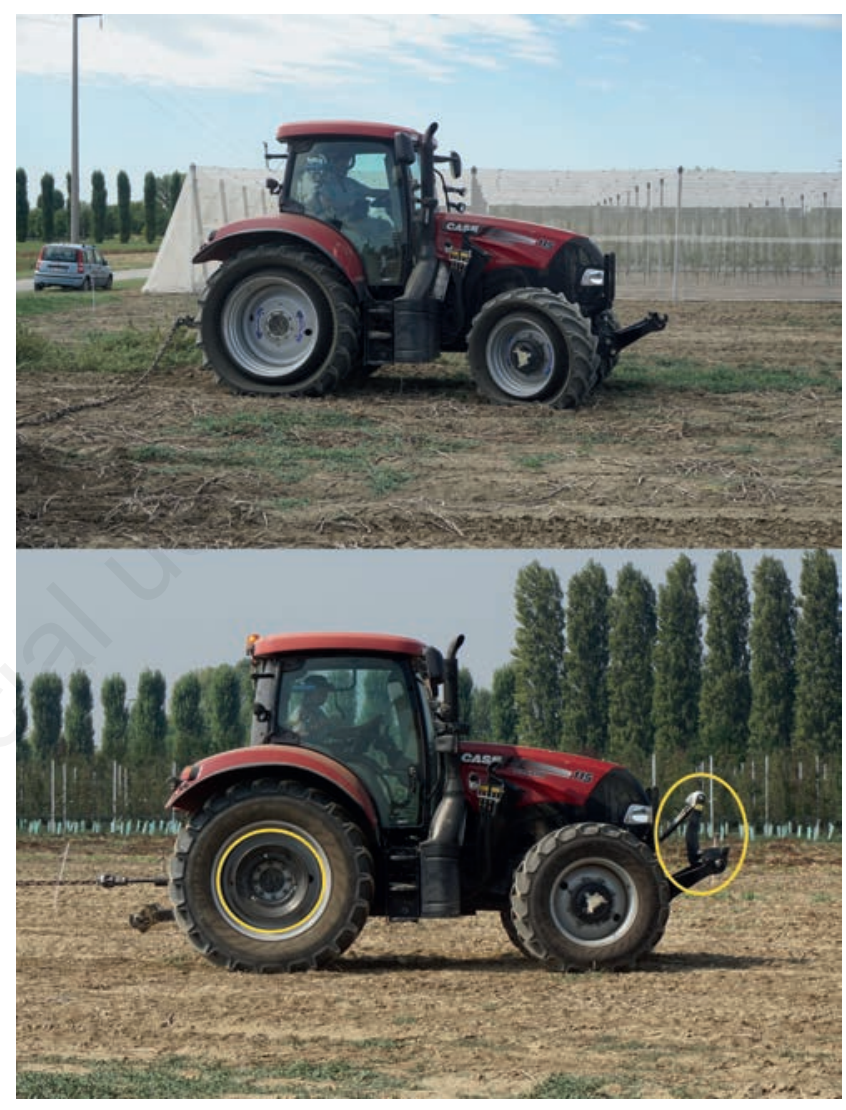

Figure 1. Tractor used for the tests at PT configuration (top) and SRT configuration (bottom). Additional ballasts are enclosed in the yellow circles.

Table 1. Tractor configurations used for the test.

Total static mass $[\mathrm{kg}]$

Front axle static mass [\%]

Rear tyres

[\%]

(20)

Front tyres

Trelleborg Pneutrac 600/65R38, 0.4 bar

Rolling radius of rear tyres (ISO, 2018) [m] Trelleborg Pneutrac 480/65R28, 0.4 bar

85

7380

46
$\operatorname{rac} 600 / 65 \mathrm{R} 38,0.4$ ba
$080 / 65 \mathrm{R} 28,0.4$ ba
.816

Tyre footprint $\left[\mathrm{m}^{2}\right]$

Rear: 0.34

Front: 0.25

Front: 0.20

Rear: 0.27

Transmission

Semi power-shift, 16 forward gears and 16 rearward gears

Lead of front wheels [\%]
SRT

44

Continental AC65 600/65R38, 1 bar

Continental AC65 480/65R28, 1 bar

0.833

OPEN $\bigcirc$ ACCESS

[Journal of Agricultural Engineering 2020; LI:1031]

[page 101] 
olutions of the rear wheel (Janulevičius et al., 2014). A higher lead was measured with the PT configuration which should contribute to increase the motion resistance during straight driving (Janulevičius et al., 2014); nevertheless, the lead for both configurations remained within the acceptable range (Ianto, 2011).

A GPS receiver (IPESpeed, IPETronik GmbH, Baden Baden, Germany) and CAN-Bus data logger (CanCase XL Log, Vector Informatik, GmbH, Struttugart, Germany) were installed on the tractor, so that the following signals were acquired: i) engine torque $\left(T_{e}\right)$; ii) engine speed $\left(\omega_{e}\right)$; iii) vehicle speed from GPS receiver $\left(V_{t}\right)$; iv) fuel temperature; v) transmission oil temperature; vi) engine coolant temperature; vii) rotational speed of rear wheels.

The engine CAN-BUS data were recorded using the method report by Molari (2013). The tractor under test towed an auxiliary tractor used as a loading unit (New Holland T7.260 with full power-shift transmission); by manipulating the throttle lever and the engaged gear, the drawbar pull could be varied. A load cell (NBC Elettronica, Sondrio, Italy) was mounted between the test tractor and the loading unit in order to measure the drawbar force (DF). Before the tests, the engine was run in idling for 30 minutes, so that the temperature of the engine coolant, transmission oil and fuel were stabilized. Thus, the influence of their variations on the results was minimized. Drawbar tests were carried out on both tractor configurations using the constant draught test procedure, since it leads to lesser data scatter (Upadhyaya et al., 1988). Thus, the drawbar pull was gradually increased from the unloading condition to that where the drawbar pull was limited by the engine power or the tractor slip exceeded 50\%. Each drawbar pull level was maintained for a running length of $20 \mathrm{~m}$ after its stabilization in order to get a steady-state condition. Each test run was carried at a specific gear and repeated for three different gears. In the two lowest gears, the tractor operated in a limited slipping condition, so that the maximum drawbar pull could be measured, while at the highest gear, the tractor could deliver the maximum drawbar power. With these gears, PT reached the maximum speeds of 4.2 , 6.5 and $9.7 \mathrm{kmh}^{-1}$, while SRT reached 4.3, 6.7 and $9.9 \mathrm{kmh}^{-1}$. Consequently, the maximum speeds of PT were from 2.6 up to
$3.4 \%$ lower than SRT on the tested gears and in unloaded conditions. During the tests, the tractor was run on a straight stretch, at full throttle, with the differential lock and front-wheel drive engaged. The differential lock was engaged to ensure the same slip of the driving wheels on the same axle.

The tests were repeated on three different soils: i) soil $U L$ : untilled loam soil mixed with residuals of the previous crop (potato); the average soil moisture content on the day of the test was $13 \%$ (plastic and liquid limit of the soil are $18 \%$ and $29 \%$, respectively); ii) soil $T L$ : loam soil which was tilled 30 days before the test with a disk plough; the average soil moisture content on the day of the test was $16 \%$ (plastic and liquid limit of the soil are $18 \%$ and 29\%, respectively); iii) soil TCL: weathered clay loam soil, previously ploughed and harrowed; the average soil moisture content on the day of the test was $21 \%$ (plastic and liquid limit of the soil are $22 \%$ and $36 \%$, respectively).

Plastic and liquid limits were measured according to the ASTM standard (ASTM, 2010). Cone index and bulk density could not be measured, especially on UL and TL soils. The former was too hard to penetrate with the penetrometer and core sampler, while the topsoil of the latter was too loose for both measurements. In order to maintain the soil and environmental conditions, tests in the two configurations and on each soil were carried out on the same day.

The vehicle slip $(s)$, traction ratio $(T R)$ and power delivery efficiency $(P D E)$ were calculated as the following:

$$
\begin{gathered}
s=\frac{N_{l}-N_{u l}}{N_{l}} \\
T R=\frac{D_{p}}{M g} \\
P D E=\frac{D_{p} V_{t}}{T_{e} \omega_{e}}
\end{gathered}
$$

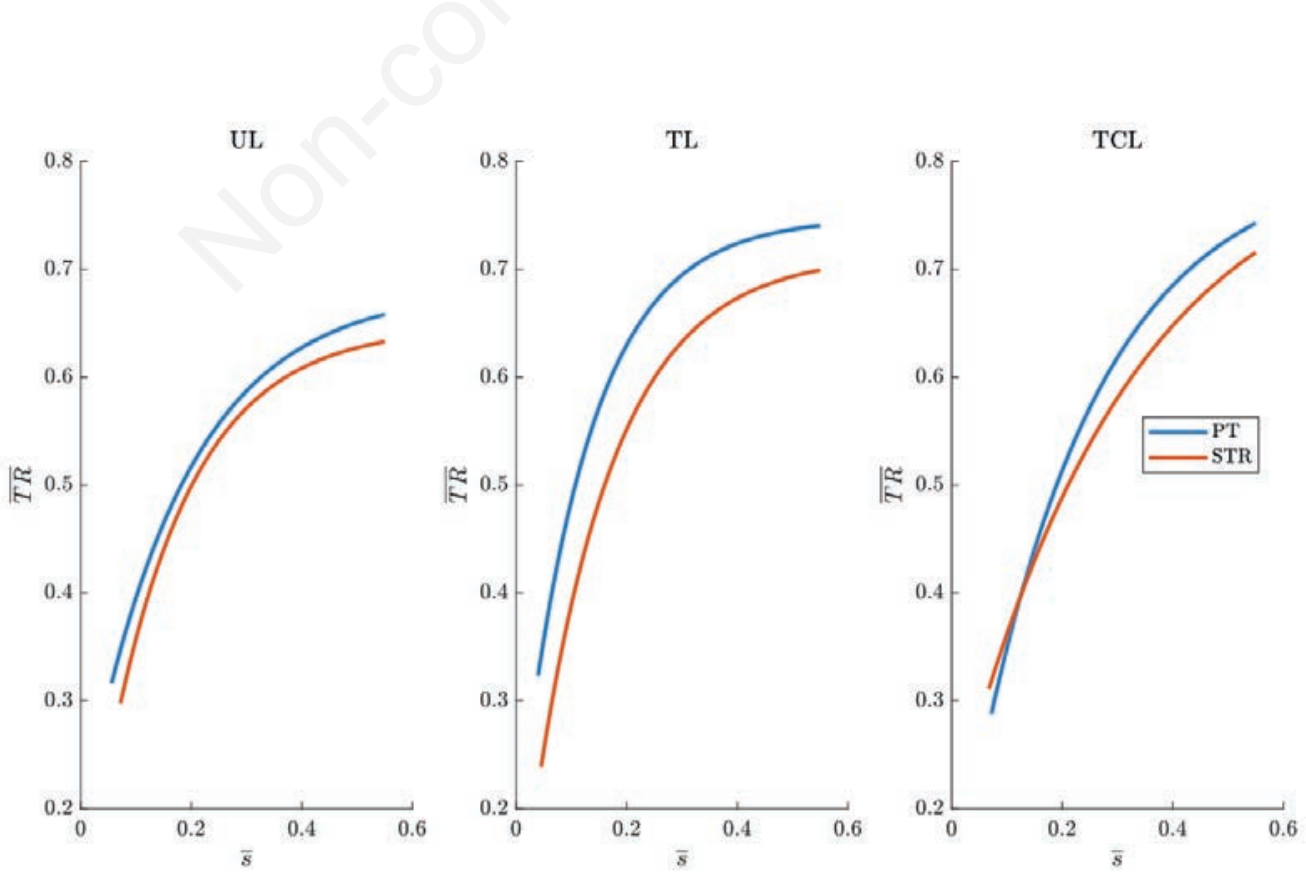

Figure 2. Experimental data with respect to the regression curves of the tractor in the PT configuration and on TL soil. 
where $N_{l}$ and $N_{u l}$ are the number of wheel revolutions in each steady condition respectively in the loaded and unloaded conditions, $M$ is tractor mass and $g$ is the gravity acceleration. $N_{l}$ and $N_{u l}$ were calculated through numerical integration of the signal of the rotational speed of the rear wheels. For the portions of all the acquired and calculated signals inside each steady condition, the average values were calculated. Overline bars are adopted in the nomenclature for the average values of each signal in the steady conditions, so that they can be distinguished from the corresponding signals (e.g. $D_{F}$ is the signal of the drawbar pull while $\overline{D_{F}}$ is the set of the average values of $D_{F}$ in steady condition portions). For a more meaningful data analysis, regressions models able to fit the trends of $\overline{T R}$ with respect to $\bar{S}, \overline{P D E}$ with respect to $\overline{T R}, \overline{T R}$ with respect $\overline{V_{t}}$ were adopted (Bashford et al., 1987):

$$
\begin{aligned}
& \overline{T R}=A\left(1-e^{-B S}\right)+C \\
& \overline{P D E}=D \overline{T R}^{2}+E \\
& \overline{T R}=F \overline{V_{t}}+G
\end{aligned}
$$

where $A, B, C, D, E, F$ and $G$ are the regression coefficients.

\section{Results and discussion}

The three regression models were tested against experimental data (an example is provided in Figure 2). $\overline{T R}$ increases with respect to $\bar{s}$ reaching a plateau at high slippage levels, because the higher is the slip and the higher is the shear stress along the longitudinal direction applied by the traction elements; which in turn permits a larger generation of the drawbar pull. $\overline{T R}$ linearly decreases with $\overline{V_{t}}$ since the tests were carried out at different engine speed levels in a portion of the engine curve where the engine torque linearly decreases with the engine speed. The trend of $\overline{P D E}$ curve with respect to $\overline{T R}$ is a convex curve, where at low drawbar levels, the efficiency is limited by the vehicle motion resistance, while at high drawbar levels, the efficiency is limited by slip losses. The regression models fit well the experimental data: data are well placed alongside the regression curves and all the Rsquared values are higher than 0.9 , except for two cases in which they are 0.81 and 0.87 (Table 2). Moreover, the R-squared values are aligned with those from previous similar studies (Bashford et al., 1993).

The trends of the regression curves of $\overline{T R}$ with respect to $\bar{s}$ for the three soil conditions are reported in Figure 3. Both configurations achieved the highest $\overline{T R}$ on TCL soil, where it was 0.74 and 0.71 for PT and SRT, respectively. On TL soil, similar values of

\begin{tabular}{|c|c|c|c|c|c|c|}
\hline & \multicolumn{2}{|c|}{$\overline{T R}$ with respect to $\bar{s}$} & \multicolumn{2}{|c|}{$\overline{P D E}$ with respect to $\overline{T R}$} & \multicolumn{2}{|c|}{$\overline{T R}$ with respect to $\overline{V_{t}}$} \\
\hline Soil & PT & SRT & PT & SRT & PT & SRT \\
\hline UL & 0.93 & 0.96 & 0.93 & 0.96 & 0.99 & 0.98 \\
\hline $\mathrm{TL}$ & 0.97 & 0.92 & 0.95 & 0.96 & 0.97 & 0.98 \\
\hline TCL & 0.81 & 0.91 & 0.87 & 0.96 & 0.95 & 0.95 \\
\hline
\end{tabular}
$\overline{T R}$ were observed to that on TCL soil. On the other hand, on UL

Table 2. R-squared values of the regression models.
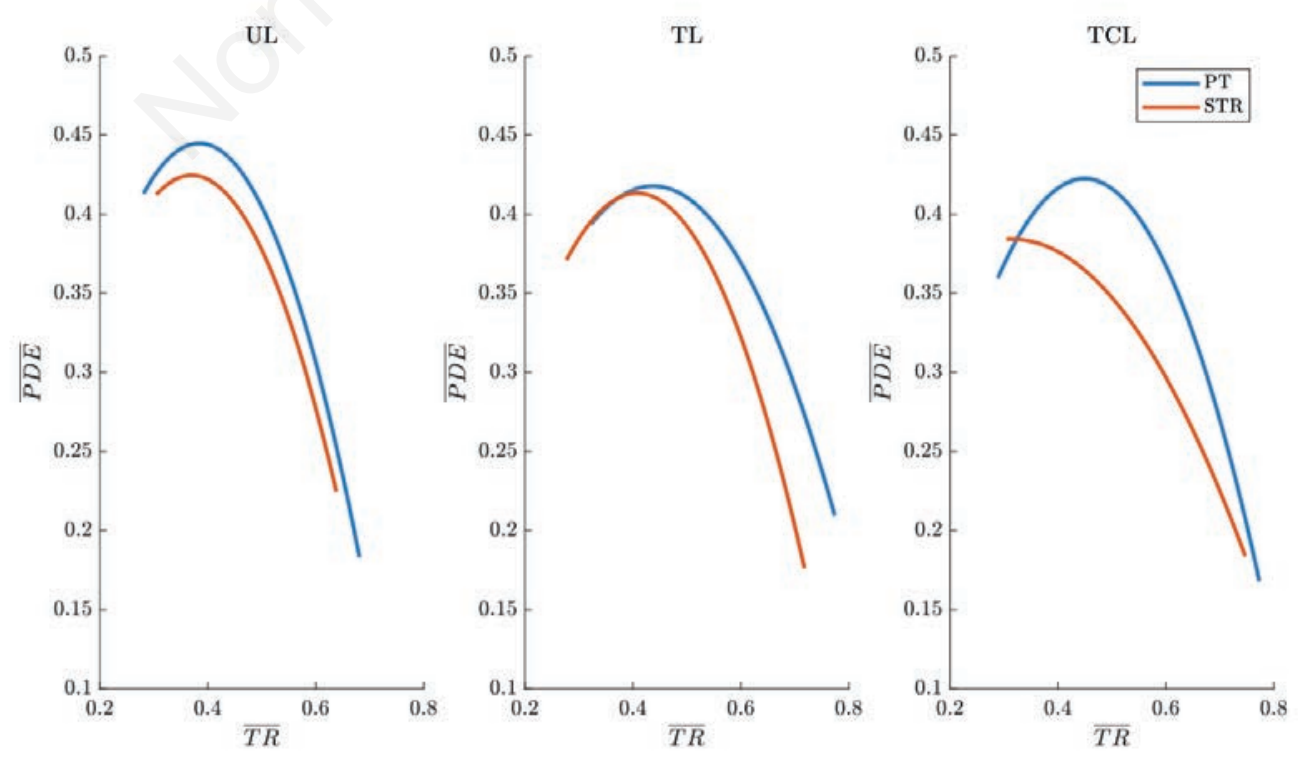

Figure 3. Regression curves of the $\overline{\mathrm{TR}}$ with respect to slip for the two tractor configurations on the three soils. 
soil, the lowest values of $\overline{T R}$ were observed, which were 0.65 and 0.63 for PT and SRT, respectively. Despite UL and TL soils falling in the same USDA classification, the higher water content of TL was beneficial for thrust development, since the moisture content is closer to the plastic limit, that is best condition for traction on soils with a high content of sand (Ali and McKyes, 1979). Moreover, TL soil was softer and, therefore, lug penetration was facilitated. Despite the higher value of the front axle lead, PT tractor developed a higher traction than SRT tractor. Indeed, the peaks of $\overline{T R}$ of PT are $4.8 \%, 5.7 \%$ and $2.8 \%$ higher than those of SRT on UL, TL and TCL soils, respectively. Consistently, at $15 \%$ of slip, $\overline{T R}$ values in the PT configuration are $1.5 \%, 16.5 \%$ and $3.7 \%$ high- er than those of SRT on UL, TL and TCL soils, respectively. On TCL soil and at slip lower than $11 \%$, SRT leaded to a higher draught, probably due to a lower rolling resistance of standard radial tyres than that of PneuTrac on this type of soil. The higher draught developed by PT is probably caused by the larger footprint of PneuTrac, which is beneficial on cohesive soils. On the other hand, the longer footprint of PneuTrac should have led to lower slip of the tractor, as it occurs for tracks (Zoz \& Grisso, 2003).

Considering the peak of each regression curve of $\overline{T R}$ with respect to $\bar{s}$, the tractor with PneuTrac was slightly less affected by soil type or condition than with the standard tyres, since $\overline{T R}$ increases up to $12 \%$ for PT, while for SRT it increases up to $14 \%$ (value are
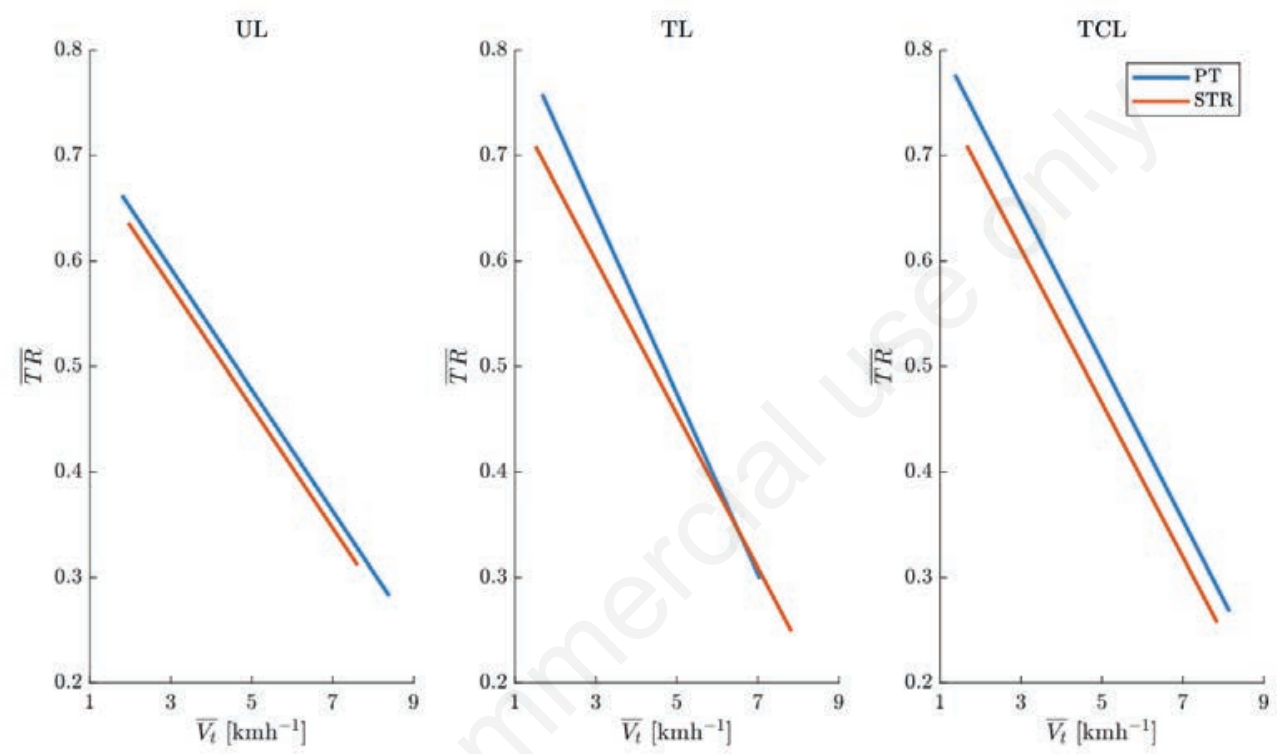

Figure 4. $\overline{T R}$ as a function of $\bar{V}_{t}$ for the two tractor configurations on the three soils.
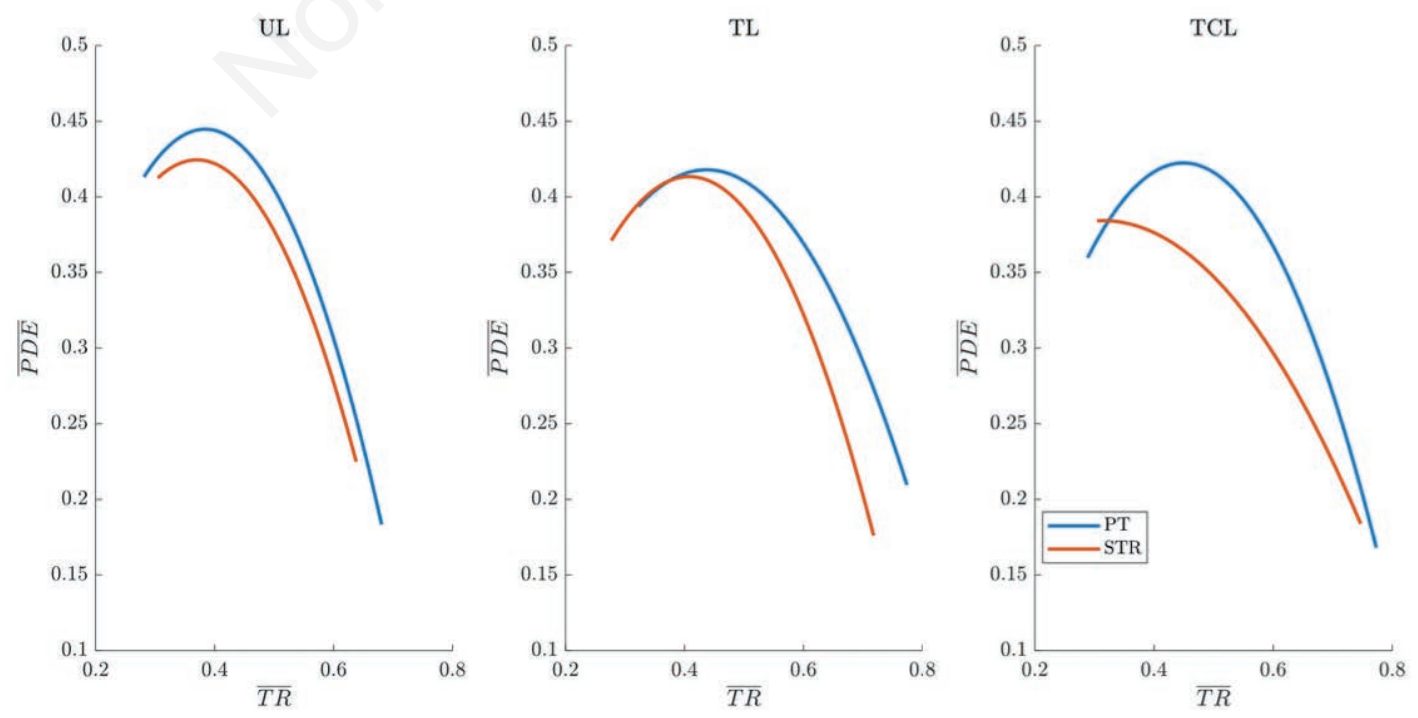

Figure 5. $\overline{P D E}$ in function of $\overline{T R}$ for the two tractor configurations on the three soils. 
given with respect to that of UL soil).

In Figure 4, the trend of the $\overline{T R}$ with respect to $\overline{V_{t}}$ is reported. PT tractor exerted a higher drawbar pull at the same speed of SRT due to the lower tyre rolling radii. This results in PT delivering a higher drawbar power at the same speed of SRT. The slopes of all the regression lines for PT are, in absolute value, slightly higher than those of SRT, due to the lower tyre rolling radius but also to the lower slip. Indeed, the largest speed gains were observed on UL and TCL soils, namely the two soils on which the advantage of $\mathrm{PT}$ in the slip efficiency is larger (Figure 3).

The trends of $\overline{P D E}$ with respect $\overline{T R}$ to on the three soils are reported in Figure 5. Peak values of $\overline{P D E}$ range from 0.39 for the SRT configuration on TCL soil up to 0.45 for the PT configuration on UL soil. The peaks of $\overline{P D E}$ curves of the PT configuration were the $7.1 \%, 5.0 \%$ and $7.7 \%$ higher on UL, TL and TCL soils, respectively. Peaks of $\overline{P D E}$ curves of SRT are located in the range of $\overline{T R}$ from 0.36 (on UL soil) up to 0.41 (on TL soil). These values are aligned with those reported in other studies for standard tyres (Jenane et al., 1996; Fancello et al., 2015). Instead, the peaks of

$\overline{P D E}$ curves of $\mathrm{PT}$ are located in the range of $\overline{T R}$ from 0.39 (on UL soil) up to 0.45 (on TCL soil). This means the most efficient condition of PneuTrac tyre is at higher draught than that of standard radial tyres and therefore they can efficiently pull closer to their limit. In addition, the curves for the PT are extended to higher

values of $\overline{T R}$ with $\overline{P D E}$ higher than 0.35 are for PT equal to 0.56 , 0.63 and 0.62 , while for SRT are equal to $0.53,0.57$ and 0.50 , on UL, TL and TCL soils, respectively. This means, PneuTrac has a range of efficient usage that is the $6 \%, 10 \%$ and $24 \%$ wider than of standard tyres.

\section{Conclusions}

In this paper, a tractor with two tyre configurations was tested to compare the tractive performance of PneuTrac tyres as opposed to that of standard radial tyres. In particular, drawbar tests were carried out on three different cohesive soils. PneuTrac provided higher drawbar pull and power delivery efficiency on all the tested soils. Consequently, PneuTrac allow a higher drawbar power to be delivered, resulting in an increased field productivity. All these benefits might be caused by the larger footprint of PneuTrac tyres, which, in turn, is provided by the greater radial flexibility of the carcass. The greater radial flexibility also allows tyres to better follow the soil profile and therefore tyre lugs penetration into the soil might be facilitated. On the other hand, the radial flexibility could also contribute to increase tyre hysteresis and, hence, tractor motion resistance too, especially on road. However, the major contribution to motion resistance on soil is soil compaction that might be reduced by using this kind of tyre. For this reason, a comparison in terms of soil compaction, motion resistance, performance evaluation during real field activities and driving comfort is recommended to better describe the field behaviour of the PneuTrac tyres.

\section{References}

Ali O.S., McKyes E. 1979. Effects on soil thrust of lug angle, length and soil consistency. Trans. ASAE 22:1294-8.

Arvidsson J., Westlin H., Keller T., Gilbertsson M. 2011. Rubber track systems for conventional tractors - Effects on soil compaction and traction. Soil Till. Res. 117:103-9.

ASTM. 2010. D4318 - Test methods for liquid limit, plastic limit, and plasticity index of soils. Available from: https://www.astm. org/Standards/D4318

Bashford L.L., Al-Hamed S., Jenane C. 1993. Effects of tire size and inflation pressure on tractive performance. Appl. Eng. Agric. 9:343-8.

Bashford L.L., Von B., Way T.R., Xiaoxian L. 1987. Performance comparisons between duals and singles on the rear axle of a front wheel assist tractor. Trans. ASABE 30:641-5.

Brassart F. 1994. Traction and Agricultural Tractor Tire Selection Studies. LSU Historical Dissertations and Theses. Available from: https://digitalcommons.lsu.edu/ gradschool_disstheses/ 5855

Fancello G., Szente M., Kovács L., Kocsis L., Szalay K., Piron E., Héritier, P. 2015. Agricultural tyre energy efficiency test method link with specific fuel consumption for measuring the efficiency of agricultural tyres under real conditions on tractors. LandTechnik. 2015:203-9.

Ianto J.G. 2011. An analysis of the interaction between the front and rear axles of a four-wheel-drive tractor, and its contribution to power delivery efficiency. Harper Adams University College, Newport, USA.

ISO. 2018. Agricultural tractor drive wheel tyres - Explanation of rolling circumference index (RCI) and speed radius index (SRI) and method of measuring tyre rolling circumference. Norm ISO R-11795:2018. International Organization for Standardization Publ., Geneva, Switzerland.

Janulevičius A., Pupinis G., Kurkauskas V. 2014. How driving wheels of front-loaded tractor interact with the terrain depending on tire pressures. J. Terramechanics 53(Suppl C):83-92.

Jenane C., Bashford L.L., Monroe G. 1996. Reduction of fuel consumption through improved tractive performance. J. Agr. Eng. Res. 64:131-8.

Kumar S., Pandey K.P., Kumar R., Ashok Kumar A. 2018. Effect of ballasting on performance characteristics of bias and radial ply tyres with zero sinkage. Measurement 121:218-24.

McKyes E. 2012. Agricultural engineering soil mechanics. Elsevier, New York, NY, USA.

Molari G., Bellentani L., Guarnieri A., Walker M., Sedoni E. 2012. Performance of an agricultural tractor fitted with rubber tracks. Biosyst. Eng. 111:57-63.

Molari G., Mattetti M., Perozzi D., Sereni E. 2013. Monitoring of the tractor working parameters from the CAN-Bus. pp 384-386 in Proc. AIIA 13. Horizons in agricultural, forestry and biosystems engineering, Viterbo, Italy.

Molari G., Mattetti M., Walker M. 2015. Field performance of an agricultural tractor fitted with rubber tracks on a low trafficable soil. J. Agricult. Engine. 46:162-6.

Novoplanski A. 2014. Patent No. US20140158268 A1.

Olesen J.E., Bindi M. 2002. Consequences of climate change for European agricultural productivity, land use and policy. Eur. J. Agron. 16:239-62.

Patel N., Slade R., Clemmet J. 2010. The ExoMars rover locomotion subsystem. J. Terramechanics 47:227-42.

Regazzi N., Maraldi M., Molari G. 2019. A theoretical study of the parameters affecting the power delivery efficiency of an agricultural tractor. Biosyst. Eng. 186:214-27.

Schjønning P., van den Akker J.J.H., Keller T., Greve M.H., Lamandé M., Simojoki A., Breuning-Madsen H. 2015. 
Chapter Five - Driver-Pressure-State-Impact-Response (DPSIR) Analysis and Risk Assessment for Soil Compaction A European Perspective. Adv. Agron. 133:183-237.

Šmerda T., Čupera J. 2010. Tire inflation and its influence on drawbar characteristics and performance - Energetic indicators of a tractor set. J. Terramechanics 47:395-400.

Turner R.J. 1993. Single, dual and triple tires and rubber belt tracks in prairie soil conditions. ASAE/CSAE International Summer Meeting. Presented at the Spokane, Washington. Spokane, Washington, DC, USA.

Upadhyaya S.K., Chancellor W.J., Wulfsohn D. 1988. Sources of variability in traction data. J. Terramechanics 25:249-72.

Zoz F.M., Grisso R.D. 2003. Traction and tractor performance. ASAE Distinguished Lecture Series n. 27. 